\title{
Denoising Baseline Wander Noise from Electrocardiogram Signal using Fast ICA with Multiple Adjustments
}

\author{
Nevi Jain \\ Department of Electronics and Comm. Engg. \\ Samrat Ashok Technological Institute \\ Vidisha, M.P., India
}

\author{
Devendra Kumar Shakya \\ Department of Bio-Medical Engg. \\ Samrat Ashok Technological Institute \\ Vidisha, M.P., India
}

\begin{abstract}
The electrocardiogram (ECG) is widely utilitarian for prognostic of heart diseases. Quality and utilization of ECG signal is affected by different noises and hence it is very difficult to measure important parameter to know the exact condition of heart. Baseline wander is one type of noise which is normally seen in ECG signal. This artifact severally limits the usefulness of recorded ECG signals and thus need to be removed for better clinical appraisal. Independent component analysis (ICA) is a statistical technique for estimating a multidimensional random vector into components that are statistically not dependent from each other. This paper proposed the implementation of fast ICA with multiple adjustments for removing baseline wander noise effect from ECG. Simulation results demonstrate that the proposed method is better in denoised the baseline wander noise from ECG signal.
\end{abstract}

\section{General Terms}

ECG, ECG Denoising, Baseline Wader Noise, Independent Component Analysis

\section{Keywords}

Electrocardiogram, Baseline Wander Noise, Variable Notch Filter, Independent Component Analysis.

\section{INTRODUCTION}

The electrocardiogram (ECG) is the recording of the electrical activity of heart which state the condition of heart is extensively utilitarian for diagnostic of heart diseases. Advising of ECG is a non-invasive technique which generally used as a preparatory diagnostic tool for cardiovascular diseases. Due to feeble non-stationary nature of ECG signal easily interfere by noise. To obtain noise free ECG signal denoising is the procedure to aloof the licit signal component from undesired signals [1]. A noise free electrocardiogram signal gives information about the electrophysiology of the heart diseases and ischemic changes that may occur [2]. Generally ECG signals frequency range is $0.05-100 \mathrm{~Hz}$ and dynamic range of ECG signal is 1-10mv. Basically ECG signal is characterized by five valleys and peaks points by the $\mathrm{P}, \mathrm{Q}, \mathrm{R}, \mathrm{S}$, and T. and its waveform is repetitive and have various bumps and parts of the waveform are designated as the P-wave, QRS-complex and T-wave, PR-segment, STsegment, PR-interval and QT-interval as given in Figure 1. Baseline wander noise is the low frequency activity in ECG signal. Due to this noise measurement of ECG parameters produce correct information is a tedious job. It can be induced by electrode changes due to perspiration, movement and respiration. Removing the baseline drift in ECG signal is most essential, if it not properly removed than some important information will be corrupted or lost. The frequency range of baseline wander noise is generally below $0.5 \mathrm{~Hz}$ which is identical as the frequency range of ST-segment [3]-[4].

Different techniques used for estimating removal baseline wander. The high pass filter used with the $0.5 \mathrm{~Hz}$ cut -off frequency. This cut-off frequency is essential for removing baseline wander and it should be preferred so that the clinical information in ECG signals remains not distorted [5]. Digital filters are generally employed to removal baseline wander noise. The cut-off frequency and phase response are two most important factors considered in digital filter designs. The utilization of linear phase filters prevents the issue of phase distortion and estimating the baseline wander [6]. Finite impulse response (FIR) filters having feed forward elements and it's usually implemented using non-recursive structures. It can have an exact linear phase. Infinite impulse response (IIR)

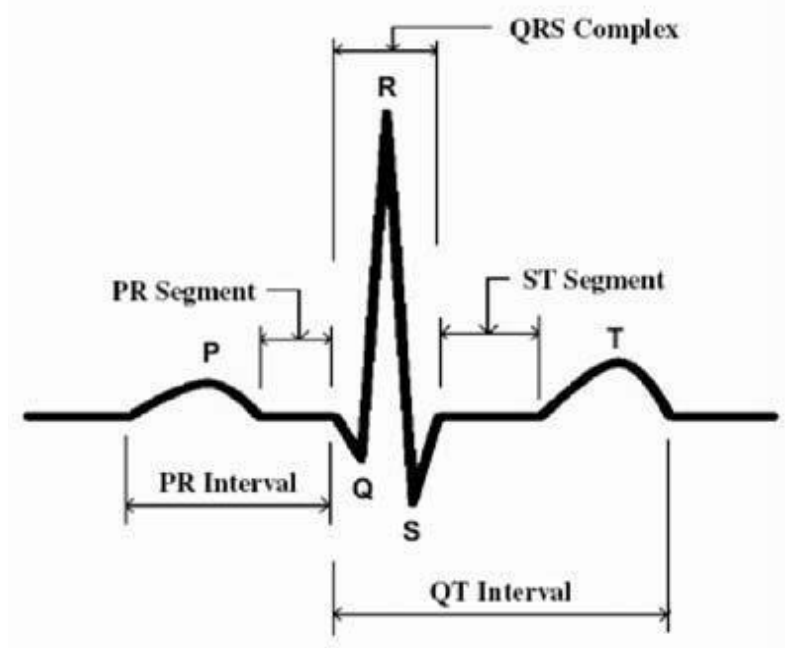

Fig 1: The ECG signal

filters are having feedback elements and IIR filters are using recursive structure. Infinite impulse response (IIR) zero phase filtering also employed removing of baseline wander [7]-[8]. These methods are use cut-off frequency for removing baseline wander and provide undistorted ECG signals.

Cubic spline curve fitting and linear spline curve fitting are the different filtering techniques which remove baseline wander by taking reference points. Cubic spline is defined as the isoelectric references points are compulsory for proper 
performance because it can create difficulties in presence of noise in ECG signals. Linear spline curve fitting method is achieve the ECG sub-signal for a single cardiac cycle which starting $60 \mathrm{~ms}$ before the P-wave and ending $60 \mathrm{~ms}$ after the Twave and then subtracting mean of sub-signal. It is most suitable for use in diagnosis procedure involving ST-segment analysis because if baseline drift is not present it does not affect the ST-segment [9]-[10]. Wavelet transforms and empirical mode decomposition (EMD) techniques are also utilized for removal baseline wander. Wavelet adaptive filters used for baseline removal from ECG signals to reduce distortion of ST-segment [11]. It is consisting with two steps. First is wavelet transform decomposes ECG signals into seven frequency bands, and second is adaptive filter [12]. Empirical mode decomposition (EMD) also offers a promising approach for baseline wanders removal [13].

Adaptive filtering is the approach for removing baseline wander. It basically used for remove DC component of ECG signal. It is also producing some distortion in ECG signal mainly in the ST-segment area [14]-[15]. Independent component analysis (ICA) is another choice of method for baseline wander removal [16]. It used to separate mutually independent component from mixed signals. Now days ICA is widely used for separating biomedical signals from mixed signal and also bio-image [17]. This approach is closely related to blind source separation (BSS) [18]. Fast ICA is another algorithm which is based on negentropy and minimum likelihood estimation [19]-[20].

Through literature survey it is observed that the estimated baseline wander by using ICA method is an approximation of the true baseline wander due to reasons discussed bellow.

- The signal yield through the multichannel can't communicate all information about the baseline wander.

- Independent component analysis used as the estimated process which may not be optimal all the time due to possibility of errors in the resulting ECG Signal.

- ICA method may be yield estimated baseline wander with number of maximum. Therefore the correct baseline wander may not be located precisely.

As such, the proposed algorithm may improve the issues allied with the non optimal multichannel signals and fast ICA method by using multiple adjustments.

The overall paper structure as follows: Section 2 describes the proposed methods. Section 3 shows the MATLAB simulations and results for the proposed algorithm and in Section 4 paper will concluded the research.

\section{Proposed Methodology}

To de-noise the baseline wander noise from the ECG signal propose algorithm implements fast ICA method to estimate

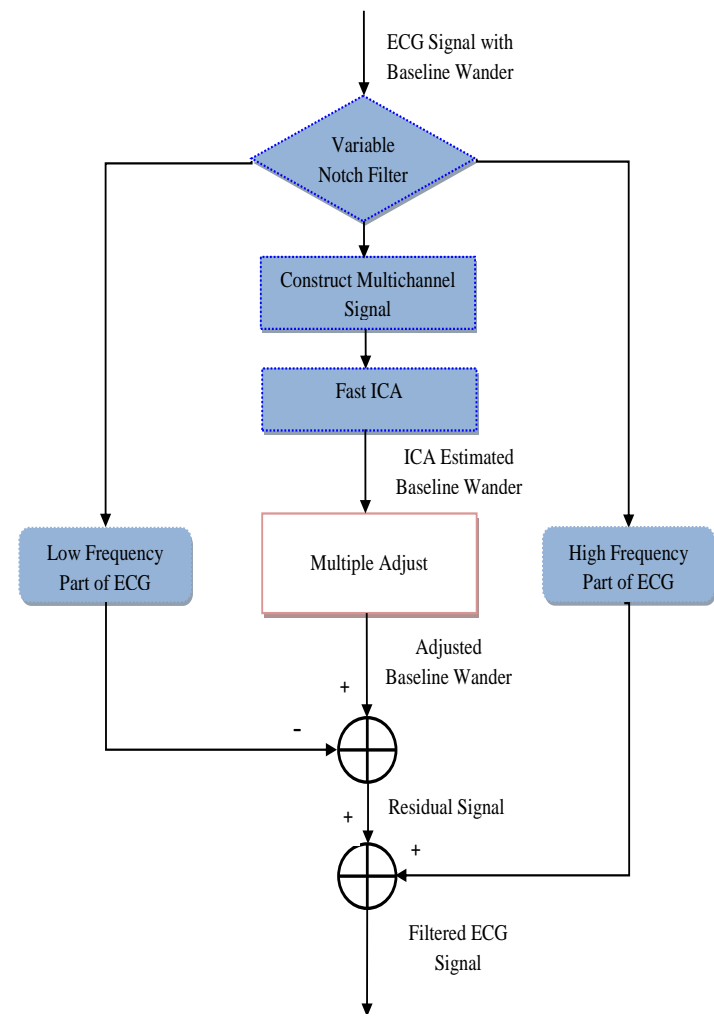

Fig 2: Schematic Diagram of Proposed Method

the baseline wander noise as reported [19]. Extracted baseline wander signal is optimized by adding an additional stage which is multiple adjustments. This method estimated the baseline wander noise signal which is eliminate it from the noisy ECG signal and yield filtered ECG signal with negligible baseline wander effect. The framework of proposed methodology's stapes as shown in Figure 2 is discussed in this section.

\subsection{Variable Notch Filter}

Contaminated ECG signal pass through variable notch filter. In the variable notch filter has two inputs are considered. One is the primary input, holding the signal and the noise and the other one is the reference input. Utilizing least mean square (LMS) principle, the reference signal is steadily approached to the noise in the primary input. At the point when the solidness is accomplished, the yield is procured through subtracting the reference input from the primary input. This kind of filter can manage inputs that are deterministic or stochastic, stationary or time-variant. The result of the adaptive noise dropping methodologies nearly wiener filter if the inputs are stationary stochastic, [15]. As to the variable notch filter, the reference signal is the one or multifixed frequencies signal, which are dealt with as the frequencies to be barred.

The transfer function of variable notch filter can be expressed as in Eq. (1)

$$
\begin{gathered}
H(z)=\frac{z^{2}-2 z \cos \left(w_{i} T\right)+1}{z^{2}-2(1-k) z \cos \left(w_{i} T\right)+(1-k)} \\
\text { and } \quad k=N \partial C^{2} / 4
\end{gathered}
$$

Where $\mathrm{N}$ is the number of stages of taped delay line of variable notch filter, $w_{i}$ is the interference frequency. If the parameter $\mathrm{N}$ is set to be fixed then this filter can be adjusted by adaptive constants $\partial$ and $\mathrm{C}$ to adopt a desired bandwidth. 


\subsection{Construct Multichannel Signal}

The ICA method required the multichannel signal as the input [16] - [20]. To use ICA for the de-noising the ECG signal, the filtered output yield from the variable notch filter is converted into multichannel ECG signal. This multichannel signals are constructed by delaying the single-channel signal which are delayed 10 sample points. To address this issue in the proposed system, an efficient methodology was made in which postponed forms of the ECG are stacked to structure the multichannel signal.

\subsection{Fast Independent Component Analysis (ICA)}

The constructed multichannel ECG signal is passes through the fast ICA algorithm to estimate the baseline wander noise signal with two sources and two sensors, ICA can be formulated as Eq. (3), $\mathrm{x} 1(\mathrm{t})$ and $\mathrm{x} 2(\mathrm{t})$ are linear combinations of the sources s1 (t) and s2 (t) [19].

$$
\begin{aligned}
& \mathrm{x}_{1}(\mathrm{t})=\mathrm{a}_{11} \mathrm{~s}_{1}(\mathrm{t})+\mathrm{a}_{12} \mathrm{~s}_{2}(\mathrm{t}) \\
& \mathrm{x}_{2}(\mathrm{t})=\mathrm{a}_{21} \mathrm{~s}_{1}(\mathrm{t})+\mathrm{a}_{22} \mathrm{~s}_{2}(\mathrm{t})
\end{aligned}
$$

The objective of the problem is to recover the unknown signal $\mathrm{s}_{1}(\mathrm{t})$ and $\mathrm{s}_{2}(\mathrm{t})$, from the mixed signal $\mathrm{x}_{1}(\mathrm{t})$ and $\mathrm{x}_{2}(\mathrm{t})$ without the known information mixing process coefficients $\mathrm{a}_{11}, \mathrm{a}_{12}, \mathrm{a}_{21}$ and $\mathrm{a}_{22}$. Where unknown signals $s_{1}(t)$ ) and $s_{2}(t)$ are statistically independent. The formal definitions of the ICA can be formulated as in Eq. (4) for multiple sources and signals [20].

$$
\mathrm{x}_{\mathrm{i}}(\mathrm{t})=\mathrm{a}_{\mathrm{i} 1} \mathrm{~s}_{1}(\mathrm{t})+\mathrm{a}_{\mathrm{i} 2} \mathrm{~s}_{2}(\mathrm{t})+\cdots+\mathrm{a}_{\mathrm{in}} \mathrm{s}_{\mathrm{n}}(\mathrm{t}) \quad \mathrm{i} \in[1, \mathrm{n}]
$$

Where $\mathrm{n}$ is the number of sources and mixture sources. This formula can also be expressed in matrix form as in Eq. (5)

$$
X=A_{n \times n} \cdot S
$$

Where $\mathrm{X}$ and $\mathrm{S}$ is the column matrices of mixture signal and sources respectively, and $A_{n \times n}$ is the mixture coefficients matrix. The solution of ICA can be achieved when distribution of the sources diverges from gaussianity. The deviation from gaussianity can be determined using measures such as negentropy. Nongaussianity can be measured by the approximation to negentropy as in Eq. (6)

$$
J(y) \alpha(E\{G(y)\}-E\{G(v)\})^{2}
$$

Where $\alpha$ represents proportionality, $\mathrm{v}$ is a gaussian variable with zero mean and unit variance, due to this $\mathrm{E}\{\mathrm{G}(\mathrm{v})\}$ is a constant. The non-quadratic function $\mathrm{G}(\mathrm{y})$ usually depends on the problem. The commonly used functions are expressed in Eq. (7)

$$
\begin{gathered}
\mathrm{G}_{1}(\mathrm{y})=\frac{1}{\mathrm{a}_{1}} \log \left(\cos \left(\mathrm{a}_{1} \mathrm{y}\right)\right) \\
\mathrm{G}_{2}(\mathrm{y})=-\mathrm{e}^{\left(-1 / \mathrm{y}^{2}\right)} \\
\mathrm{G}_{3}(\mathrm{y})=\mathrm{y}^{4}
\end{gathered}
$$

Where a1 $\epsilon[1 ; 2]$

Some preprocessing is necessary before applying ICA in input signal. These processes are centering and whitening, the obtained signals ought to be centered by subtracting their mean value and then they are whitened by linearly transformed to make components uncorrelated and have unit variance.
Whitening can be performed by using eigenvalue decomposition to the covariance matrix shown in Eq. (8)

$$
\mathrm{E}\left[\mathrm{y} \cdot \mathrm{y}^{\mathrm{T}}\right]=\mathrm{D} \cdot \mathrm{V} \cdot \mathrm{D}^{\mathrm{T}}
$$

Where, D and $\mathrm{V}$ are the orthogonal matrix of eigenvectors and diagonal matrix of eigenvalues respectively. Now a new variable $\mathrm{Z}$ can be represent affecter whitening by Eq. (9) and Eq. (10)

$$
\begin{gathered}
Z=D \cdot V^{1 / 2} \cdot D^{T} \cdot y \\
Z=D \cdot V^{1 / 2} \cdot D^{T} \cdot P s=\widetilde{P} s
\end{gathered}
$$

The fast and fixes point ICA is the direct extension of standard ICA. The functionality of fast and fixed point ICA is to find a way, which can maximize non-gaussianity $\mathrm{w}^{\mathrm{T}} \mathrm{x}$. The stapes of ICA algorithm are:

- Initialize $\mathrm{w}$ as a weight vector in one direction

- Weight vectors updating according to the following criterion as Eq. (11)

$$
\mathrm{w}^{*}=\mathrm{E}\left\{\mathrm{xg}\left(\mathrm{w}^{\mathrm{T}} \mathrm{x}\right)\right\}-\mathrm{E}\left\{\mathrm{xg}^{\prime}\left(\mathrm{w}^{\mathrm{T}} \mathrm{x}\right)\right\} \mathrm{w}
$$

and weight normalization is as Eq. (12)

$$
\mathrm{w}=\mathrm{w}^{*} /\left\|\mathrm{w}^{*}\right\|
$$

- Repeat above step if the weights have not converged, where $\mathrm{w}$, is the weight vector to calculate latent source $\mathrm{s}=\mathrm{w}^{\mathrm{T}} \mathrm{x}$.

\subsection{Multiple Adjustment}

The estimated multichannel baseline wander noise signal through fast ICA method may not be up to the mark as discussed in the previous section, as such the proposed multiple adjustment process may alleviate the issues related with the non-optimal construction of multi-channel signals. Before adjusting the extracted baseline drift multichannel signal is reconstructed to single channel signal. To adjust the ICA extracted baseline wander noise, this signal is correlated with the noisy ECG signal. The maximum resemblance in peaks of denoised ECG which is obtained after removing baseline wander noise with input ECG is obtained for maximum value of ' $\tau$ '. The correlation of noisy ECG signal and ECG obtained after extraction of baseline wander noise is represented by Eq. (13)

$$
C(\tau)=\sum_{-m}^{m} h(n) y(n-\tau)
$$

Where, $h(n)$ is input ECG signal, $y(n)$ is ECG signal obtained after applying ICA, $\tau$ is correlation factor which defines degree of similarity between input ECG and ECG obtained after applying ICA, $C(\tau)$ is optimized ECG signal.

\subsection{Removing Low Frequency Part of ECG Signal}

The adjusted baseline wander noise signal is now subtracted from low frequency part of the noisy ECG signal. Here center frequency for the low pass filter is taken as $0.5 \mathrm{~Hz}$. This low frequency removal process produces residual ECG signal.

\subsection{Combining High Frequency Part of ECG Signal}

The residual ECG signal is at the end is combined with the high frequency part of the ECG signal to regenerate the high frequency ECG parts which may collapse by filtering process, which yield filtered ECG signal with improved SNR and without baseline wander effect. 


\section{MATLAB Simulation}

To test the proposed algorithm for de-noising baseline wander noise form ECG signal, is simulated on MATLAB and tested with the de-noised ECG signal founded from MIT BIH Arrhythmia data base, with MATLAB generated baseline wander noise [21].

The MATLAB simulated results are presented in this section.
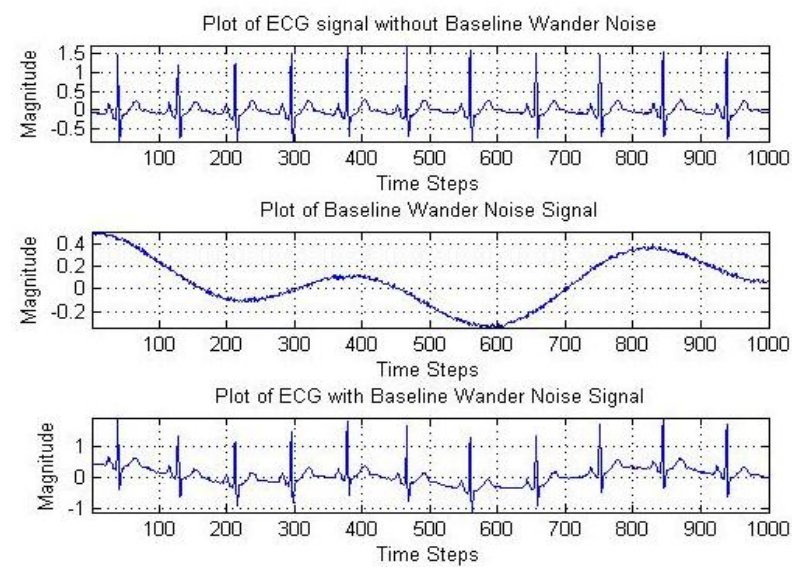

Fig 3: ECG with and without added baseline wander noise
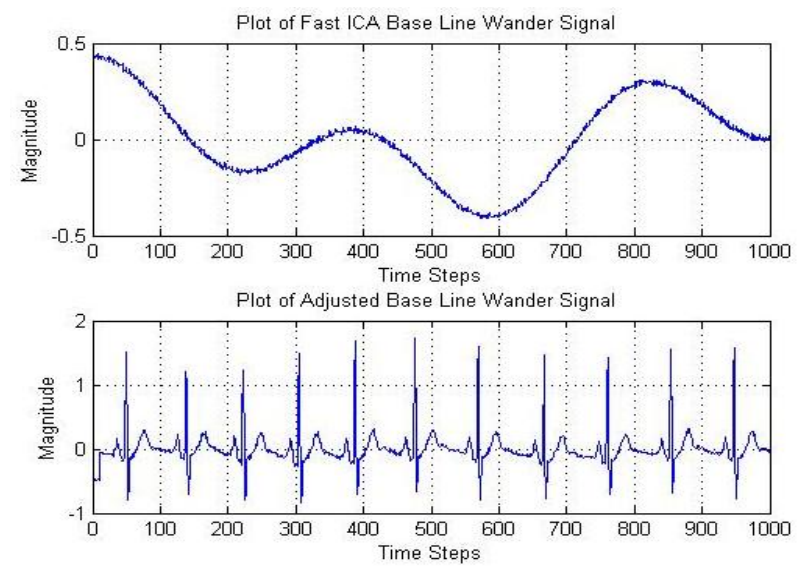

Fig 4: Comparison between estimated baseline wader noise signal after Fast ICA and adjustments
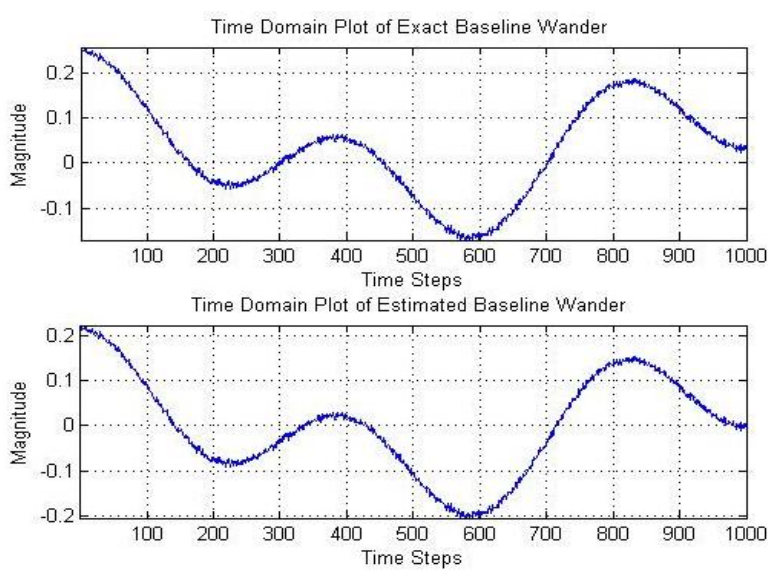

Fig.5. Comparison of errors between Matlab generated baseline drift and finally estimated baseline drift after adjustment
In Figure 3 represents plot of ECG signal without baseline drift, MATLAB generated baseline wander noise signal and ECG signal with added baseline wander noise. This noisy ECG signal is processed with the proposed algorithm.

In Figure 4 baseline drift plot after fast ICA process and after proposed multiple adjustments process is compared with each other. Form Figure 3 and 4 it is clear that the proposed multiple adjustment process estimates baseline drift signal much optimized then fast ICA. The plot of matlab generated baseline drift signal and finally adjusted baseline drift is shown in Figure 5. These figures also represents plot error signal between original ECG signal without baseline wander noise and filtered ECG signal through the proposed algorithm.
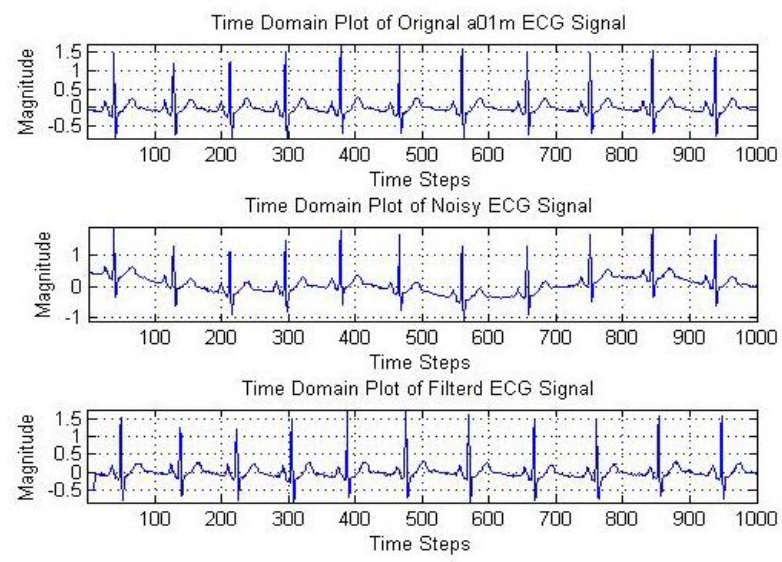

Fig 6: Filtered a01m ECG result plot
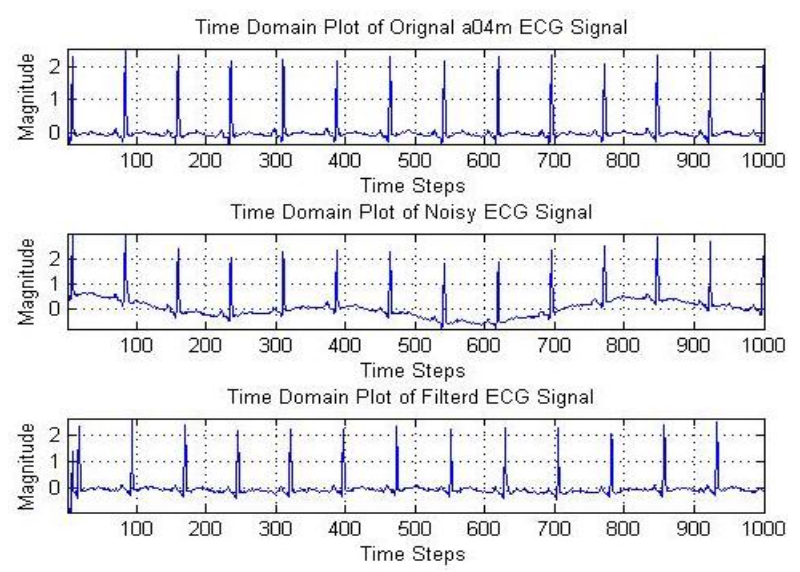

Fig 7: Filtered a04m ECG result plot
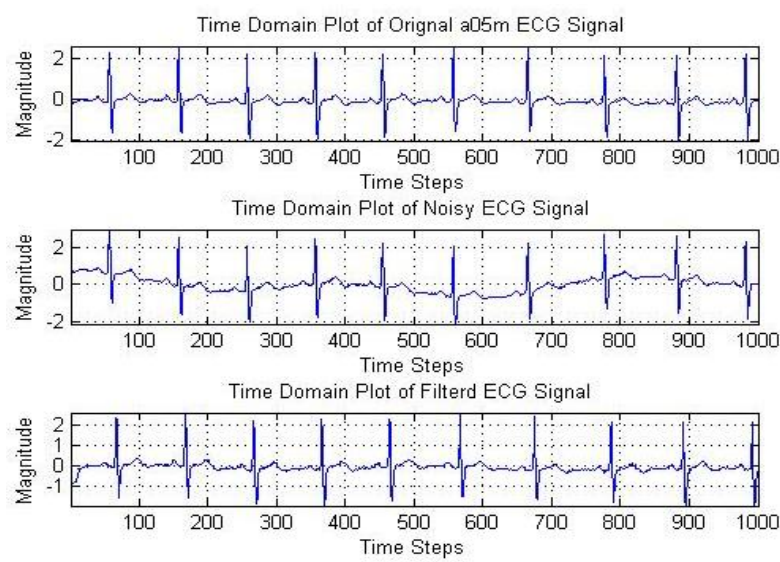

Fig 8: Filtered a05m ECG result plot 

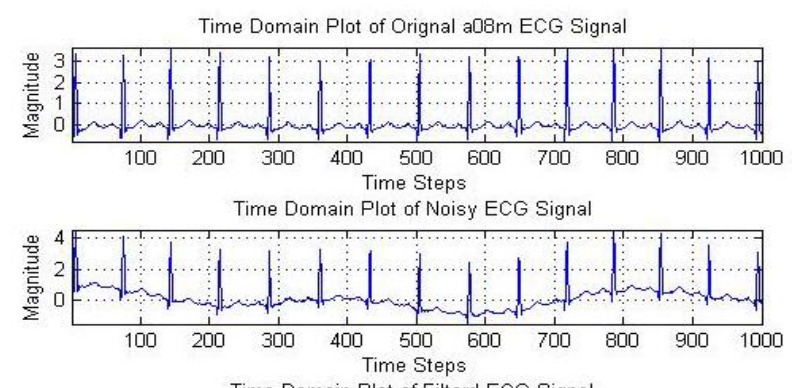

Time Domain Plot of Filterd ECG Signal

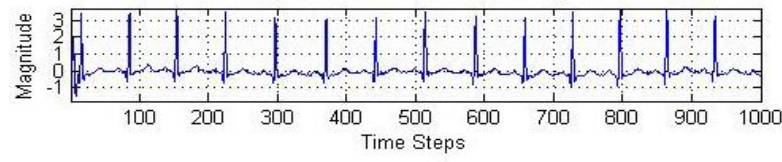

Fig 9: Filtered a08m ECG result plot

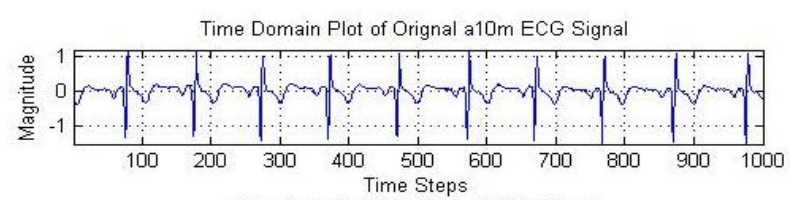

Time Domain Plot of Noisy ECG Signal

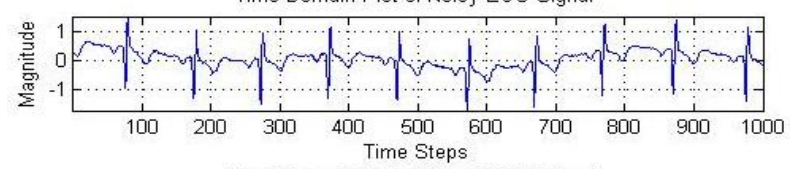

Time Domain Plot of Filterd ECG Signal

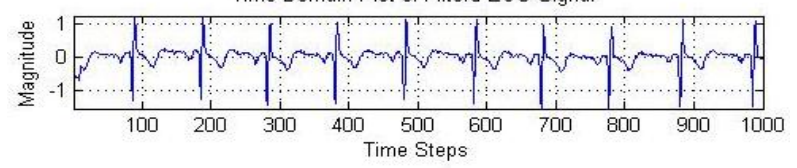

Fig 10: Filtered a10m ECG result plot

Table 1. MSE and SNROUT Results of ECG Sample Signal Using Proposed Algorithm

\begin{tabular}{|c|c|c|c|c|}
\hline \multirow{2}{*}{$\begin{array}{c}\text { ECG } \\
\text { DATA } \\
\text { SIGNAL }\end{array}$} & \multicolumn{2}{|c|}{$\begin{array}{c}\text { NOISY ECG } \\
\text { SIGNAL }\end{array}$} & \multicolumn{2}{c|}{$\begin{array}{c}\text { FILTERED ECG } \\
\text { SIGNAL }\end{array}$} \\
\cline { 2 - 5 } & MSE & $\begin{array}{c}\text { SNR } \\
\text { (db) }\end{array}$ & MSE & $\begin{array}{c}\text { SNR } \\
\text { OUT } \\
(\mathbf{d b})\end{array}$ \\
\hline $\mathbf{a 0 1 m}$ & 0.1732 & -4.2511 & 0.1290 & -2.9690 \\
\hline $\mathbf{a 0 4 m}$ & 0.3410 & -4.4284 & 0.2515 & -3.1066 \\
\hline $\mathbf{a 0 5 m}$ & 0.5041 & -4.2446 & 0.3676 & -2.8736 \\
\hline $\mathbf{a 0 8 m}$ & 0.9988 & -4.5996 & 0.7522 & -3.3685 \\
\hline $\mathbf{a 1 0 m}$ & 0.2047 & -4.2476 & 0.1529 & -2.9810 \\
\hline
\end{tabular}

This algorithm is tested for five ECG samples with added baseline wander noise signal and there results are shows from Figure 6 to 10, which show the original data of ECG signal, ECG signal with added baseline drift and filtered ECG signal through proposed algorithm.

Table 1 represents the output SNR and Mean Square Error (MSE) for all five ECG samples calculated by proposed algorithm. From table 1 inference drawn is that minimum MSE is obtained for a01m ECG data signal and maximum SNR is obtained for a08m ECG data signal.

\section{CONCLUSIONS}

The present paper demonstrates the ECG baseline wander denoising through the fast ICA with multiple adjustments to improve the baseline drift estimation of ICA algorithm. This algorithm reduces MSE as minimum MSE is obtained for a01 signal is 0.129 and maximum SNR is obtained for a08m ECG signal is -3.3685 . This method is very flexible method due to the multiple adjustments for the optimization of baseline wader noise signal.

\section{ACKNOWLEDGMENT}

The authors would like to express their sincere thanks to Dr. S. N. Sharma, Head of the Department of Electronics and Communication Engineering, for his kind support for this research work. Authors would also like to express their sincere thanks to all the authors of the reference papers and all the faculty members of the department.

\section{REFERENCES}

[1] Joshi, S. L., Vatti R. A., and Tornekar, R. V. 2013. A Survey on ECG Signal Denoising Techniques. International Conference on Communication Systems and Network Technologies. 60-64.

[2] Das, M.K., and Ari, S. 2013. Analysis of ECG signal denoising method based on S-transform. IRBM. Vol. 34. Issue 6. 362-37.

[3] Gurumurthy, S., and Valarmozhi. 2013. System Design for Baseline Wander Removal of ECG Signals with Empirical Mode Decomposition Using Matlab. International Journal of Soft Computing and Engineering (IJSCE). Vol. 3. Issue 3.

[4] Jane, R., Laguna, P., Thakor, N.V., and Caminal, P. 1992. Adaptive baseline wander removal in the ECG: comparative analysis with cubic spline technique. Proceedings of Computers in Cardiology, Durham, NC. 143-146.

[5] Chouhan, V. S., and Mehta, S. S. 2007. Total removal of baseline drift from ECG signal. In Proceedings of International Conference on Computing: Theory and Applications (ICCTA '07). 512-515.

[6] Van Alst'e, J. A., Van Eck, W., and Herrmann, O. E. 1986. ECG baseline wander reduction using linear phase filters. Computers and Biomedical Research. Vol. 19. No. 5. 417-427.

[7] Kaur, M. and Seema. 2011. Comparisons of Different Approaches for Removal of Baseline Wander from ECG Signal. International Journal of Computer Applications (IJCA). Vol.5. 30-34.

[8] Harting, L. P., Fedotov, N. M., and Slump, C. H. 2004. On baseline drift suppressing in ECG-recordings. In Proceedings of the IEEE Benelux Signal Processing Symposium. 133-136.

[9] Hyvärinen, A., and Oja, E. 2000. Independent Component Analysis: Algorithms and Applications. In Neural Network. Vol. 13. Issues 4-5. 411-430.

[10] Meyer, C. R., and Keiser, H. N. 1977. Electrocardiogram baseline noise estimation and removal using cubic splines and state-space computation techniques. In Computers and Biomedical Research. Vol. 10. No. 5. 459-470. 
[11] Park, K. L., Lee, K. J., and Yoon, H. R. 1998. Application of a wavelet adaptive filter to minimize distortion of the ST-Segment. Medical and Biological Engineering and Computing. Vol. 36. 581-586.

[12] Blanco-Velasco, M., Weng, B., and Barner, K. E. 2008. ECG signal denoising and baseline wander correction based on the empirical mode decomposition. Computers in Biology and Medicine. Vol. 38. 1-13.

[13] Kabir, Md. A., and Shahnaz, C. 2012. Denoising of ECG signals based on noise reduction algorithms in EMD and wavelet domains. Biomedical Signal Processing and Control. Vol.7. 481- 489.

[14] Laguna, P., Jane, R., and Caminal, P. 1992. Adaptive Filtering of ECG Baseline Wander. Engineering in Medicine and Biology Society, International Conference of the IEEE. Vol.14.

[15] Widrow, B., Glover, J. R., and McCool, J. M. 1975. Adaptive noise cancelling: principles and applications. Proceedings of the IEEE. Vol. 63. No. 12. 1692-1716.

[16] Barati, Z., and Ayatollahi, A. 2006. Baseline wandering removal by using independent component analysis to single-channel ECG data. Proceedings of International Conference on Biomedical and Pharmaceutical Engineering (ICBPE '06). 152-156.

[17] Nimitha, U. and Supriya, P. 2011. Independent Component Approach for the Analysis of ECG Signals. Proceedings of the IEEE. 1-5.

[18] Milanesi, M., Vanello, N. and Positano, V. 2005. Frequency domain approach to blind source separation in ECG monitoring by wearable system. in Proceedings of Computers in Cardiology. 767-770.

[19] Luo, Y., Hargraves, R. H., Belle, A., Bai, O., Qi, X., Ward, K. R., Pfaffenberger, M. P., and Najarian, K. 2013. A hierarchical method for removal of baseline drift from biomedical signals: application in ECG analysis. The Scientific World Journal. 1-10.

[20] Hyvarinen. Fast and robust fixed-point algorithms for independent component analysis. IEEE Transactions on Neural Networks. Vol. 10. No. 3. 626-634.

[21] US Patent, 'US5318036', Hewlett Packard. 